\title{
Tau tubulin kinase 2 is required to initiate mammalian ciliogenesis
}

\author{
S Goetz, KV Anderson \\ From First International Cilia in Development and Disease Scientific Conference (2012) \\ London, UK. 16-18 May 2012
}

Despite the critical importance of cilia in development and disease, the biochemical pathways that initiate ciliogenesis at the mother centriole are not well understood. Here we describe the identification of a serine-threonine protein kinase, Ttbk2, as an essential component of the ciliary initiation pathway. The mouse mutant bartleby (bby) was isolated in a genetic screen in our lab based on phenotypes consistent with a severe disruption in Hh signaling, and immunostaining and scanning electron microscopy revealed that bby mutants lack cilia. The bby phenotype is caused by a nonsense mutation in the gene encoding Tau Tubulin Kinase 2 (Ttbk2), truncating the protein within the kinase domain. Independent studies have shown that human mutations in Ttbk2 are associated with the human neurodegenerative disorder spinocerebellar ataxia type 11 . We show that Ttbk2::GFP localizes to the distal mother centriole in cells prior to ciliary axoneme extension, and to the transition zone between the basal body and axoneme in ciliated cells. In Ttbk $2 b b y$ mutant cells, components of the IFT complexes, such as IFT88 and IFT140, are not recruited to the transition zone. Thus Ttbk2 acts at a step upstream to IFT in the process of cilia formation and is a critical component of a pathway regulating the initiation of ciliogenesis. Although several kinases have roles in ciliogenesis, Ttbk2 is thus far the only kinase found to have an essential role in cilia formation. Moreover, identification of Ttbk2 substrates and upstream regulators has the potential to reveal other regulators of this important step in ciliogenesis.

Published: 16 November 2012

* Correspondence: goetzs@mskcc.org

Sloan-Kettering Institute, New York, USA
doi:10.1186/2046-2530-1-S1-018

Cite this article as: Goetz and Anderson: Tau tubulin kinase 2 is required to initiate mammalian ciliogenesis. Cilia 2012 1(Suppl 1):018.
Submit your next manuscript to BioMed Central and take full advantage of:

- Convenient online submission

- Thorough peer review

- No space constraints or color figure charges

- Immediate publication on acceptance

- Inclusion in PubMed, CAS, Scopus and Google Scholar

- Research which is freely available for redistribution

Submit your manuscript at www.biomedcentral.com/submit

\section{() Biomed Central}

